\title{
USE OF WEB 2.0 IN CULTURAL HERITAGE
}

\author{
Lola García-Santiago and María Dolores Olvera-Lobo \\ Department of Information and Communication Sciences, University of Granada, Spain \\ Faculty of Translation and Interpreting. Calle Buensuceso 11 Despacho 11. Granada (Spain) 18071
}

\begin{abstract}
As example of digital humanities in this paper the presence of institutional websites for heritage assets on relevant social media and Web 2.0 tools was analysed (social tools, Wikipedia and blogs). It was studied if new forms of cultural communication are being used as actual communication ways. The presence of institutional profiles was analysed on the following social networks: Facebook, Twitter, Google+, YouTube, Vimeo, Instagram, Flickr, Pinterest, TripAdvisor and FourSquare. Furthermore, it was identified the presence of heritage assets in the "Google Arts \& Culture" project of the Google Cultural Institute as a new digital cultural resource. Results heterogeneous have been obtained and have been detected lacks in the adaption web 2.0. Wikipedia is the web 2.0 tool more used and only the $50 \%$ of the cases use Facebook or Twitter -most of them governmental institutions which manage cultural assets-. Image-based social networks are used but not in a usual way.
\end{abstract}

\section{KEYWORDS}

Digital Humanities, Web 2.0, World Cultural Heritage, Google Arts \& Culture, Social Networks

\section{INTRODUCTION}

The concept denominated by the sociologist Zygmunt Bauman (2003) as liquid modernity is in constant evolution and adaptation to change. In the context we refer to in this work, the need for constant access to information demands the usage and appropriate management of the tools available on the Web 2.0 for a dissemination of content in accordance with the times we live in. Social media sites disseminate and share content in a community manner in the hope of feedback on the part of members. Institutions wishing to increase the expansion of the information they produce, along with their own presence and visibility, need to integrate with this group. It is for this reason that there is a progressive aware of what good digital identity management implies for improving visibility and facilitating participation and contact with users.

From the perspective of digital humanities, it is worth asking how the dissemination of information on cultural heritage is being implemented. In this regard, initiatives such as Google Arts \& Culture, proposed by the Google Cultural Institute (2020) and to which reference will be made further on, are of great interest. Likewise, with increasing frequency, the bodies themselves are responsible in one way or another for the dissemination of information on heritage, mainly public bodies or non-profit governmental cultural institutions, which endeavor to create a digital identity and reinforce the on-line presence of cultural assets (García-Santiago and Olvera-Lobo, 2018). Social media in general and particularly social networks, unquestionably have a prevailing role in this task.

Therefore, given the enormous repercussion that heritage has on the cultural identity of towns, economic sectors involved in local development, or tourism, among many other factors, it is essential to be aware of the influence of the aforementioned communication structures (Jiménez-Orellana, 2016 and Rojas Castro, 2016). The analysis of how the digital identity of our heritage assets and the dissemination of information on them contributes to better knowledge of the function and optimum usage of these new technological and communicational tools.

Web 2.0 is the name given to the group of applications that take into account the effects of network interaction and exploit them via the participation and information generated on the part of users and consumers. That is, the Web is seen as a large platform where you can participate, share, interact and cooperate via programs executed online and used by multiple users at the same time (Castelló Martínez, 2010; Viñarás Abad, 2010;O’Reilly, 2005). 
Web 3.0 takes it one step further. This term, which has not had much effect up to now, puts forward a more commitment concept of the Internet, in the sense that it advocates the common good and sustainable development of the Web. The idea behind Web 3.0 is therefore to extend the benefits of Web 2.0 to more people -encouraging accessibility and the use of mobile devices-, more functions and more applications, affording it more human meaning and a greater social and environmental impact.

\section{METHODOLOGY}

The study sample is comprised of 45 assets recognized by Unesco as World Heritage located in Spain, the European nation with the highest number of assets distinguished as such, until March 2018. In a manual processing, the institutional website for each of the heritage assets was identified, via consultations made via Google web search. The queries were considered from the denomination used by Unesco for each heritage asset, such as for example "Silk Exchange of Valencia" - and/or a variation on its name, if this turned out to be more recognizable. This is the case, for example, of "Pre-Romanesque Asturian" instead of "Monuments of Oviedo and the Kingdom of the Asturias", which is the denomination adopted by Unesco. The name of a heritage asset therefore frequently covers various monuments, places or assets. Thus, there are different cases of heritage assets with a "collective denomination" such as the "Historic City of Toledo" and the Monastery and Site of the Escorial, Madrid". In these examples it was necessary to locate and compile as many websites as subdivisions that resulted from said denomination. Commercial pages were excluded from the sample, and we selected those originating from some type of preferably public, private or mixed entity that guaranteed its legitimacy. As a result of this phenomenon, of the 45 assets classified by Unesco as cultural World Heritage, we analyzed 71 URLs of institutional websites that showed contents on the asset in the form of a simple web page or homepage, or in the form of a group of them for presenting different aspects of the heritage asset in a single documental unit called an institutional domain. If the group of pages is extremely voluminous, it may be an independent website under a domain such as .org, .es, etc. and with a structure such as, for example, $w w w . w e b s i t e . t o p l e v e l d o m a i n$. This step requires a human review and consequently it is not possible to be done automatically.

The presence of institutional profiles was analyzed on the following social networks: Facebook, Twitter, Google+, YouTube, Vimeo, Instagram, Flickr, Pinterest, TripAdvisor and FourSquare. Furthermore, where appropriate, we studied the existence of links from the heritage asset websites to these social profiles. In addition, Wikipedia entries and the existence of blogs relating to these cultural assets were identified, along with their presence on the Google Institute project.

The social networks - the most popular social media type- the analysis focused on were Facebook, Twitter, Google+, YouTube, Vimeo, Instagram, Flickr, Pinterest, TripAdvisor and FourSquare. On the one hand, we checked to see whether the institutional URLs of the cultural assets had a profile on these networks. In addition, we have explored if web pages of the sample are linked more if they have official profile on any of the different social networks and media of the cultural assets.

Furthermore, entries corresponding to heritage assets in the study sample were identified on Wikipedia, another of the social mediums that enjoys extensive acceptance and popularity amongst Web 2.0 users. We also analysed blogs. In this manner, in addition we were able to specify the use made of this Web 2.0 tool. We also determined which structure and content characteristics these blogs presented.

In parallel, we carried out searches in Google Arts \& Culture from the denomination of each asset and identified the type of information collected in this Google Cultural Institute tool.

\section{RESULTS}

The data obtained from an analysis of the presence of institutional web pages of the sample heritage assets on the different social media sites reveal, overall, that $13 \%$ of the web pages considered do not yet have a Web 2.0 presence, even where the institution responsible for the management of the heritage asset does. This $13 \%$ of URLs correspond to 7 assets, that is, $15.6 \%$ of the sample, which are not totally or partially represented on any social media considered in the study. By way of example, this is the case for the "Historical City of Caceres", which, as the collective denomination it is, has 4 URLs that represent it. However, only one of its 
URLs, -that corresponding to a part of this heritage asset, specifically the Church of san Francisco Javier-, is found to be represented on the Web 2.0 (in other words, a mere $25 \%$ of the total information on the heritage asset in question).

Figure 1 shows the results relating to the presence on social networks of the different institutional websites. From the social media used, $68 \%$ of the assets included in the sample have a presence on Wikipedia, followed by the social networks Facebook and Twitter. In contrast, the least used social networks are those that collect opinions and ratings both on the part of users and of any other person who wishes to express their comments, as in the case of TripAdvisor and Foursquare. The use of audiovisual formats is even more in the minority on the part of institutions on social networks such as Pinterest and Vimeo.

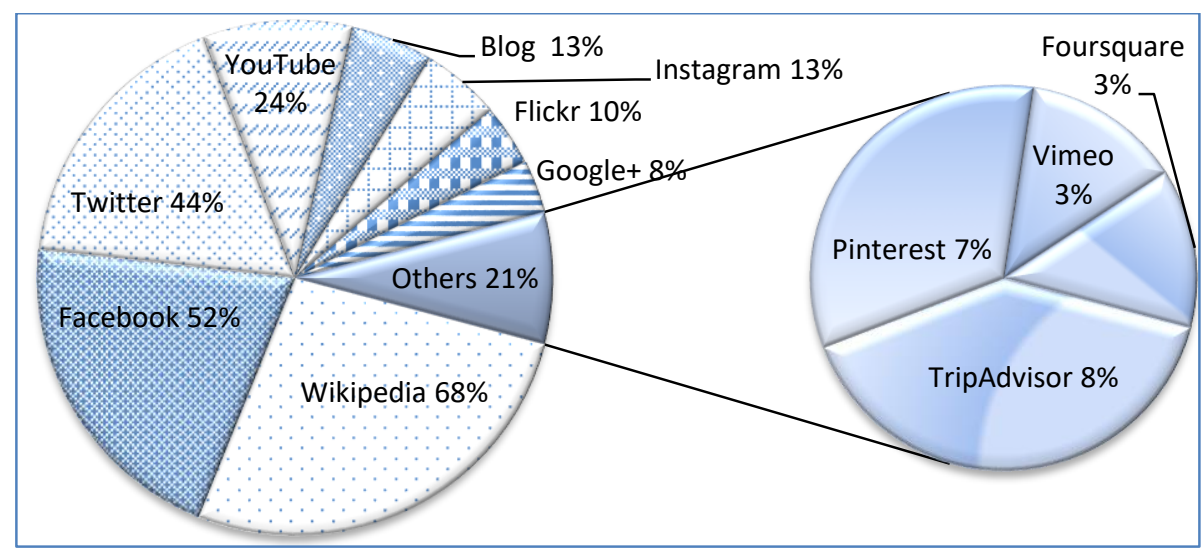

Figure 1. Web 2.0 presence of World Heritage (\% of URLs)

As mentioned, on occasions there are more than one URL for a heritage asset where it is a monument complex containing various elements, that is, it has a collective denomination. For this reason, as a complement to the previous analysis, we considered the total or partial Web 2.0 presence of the heritage assets themselves (Figure 2) regardless of the number of URLs of each asset which is shown in former figure 1.

Again, the social media site most used by the World Heritage assets included in our study sample is Wikipedia, with $73 \%$ of the cases with a presence on this collaborative encyclopedia.

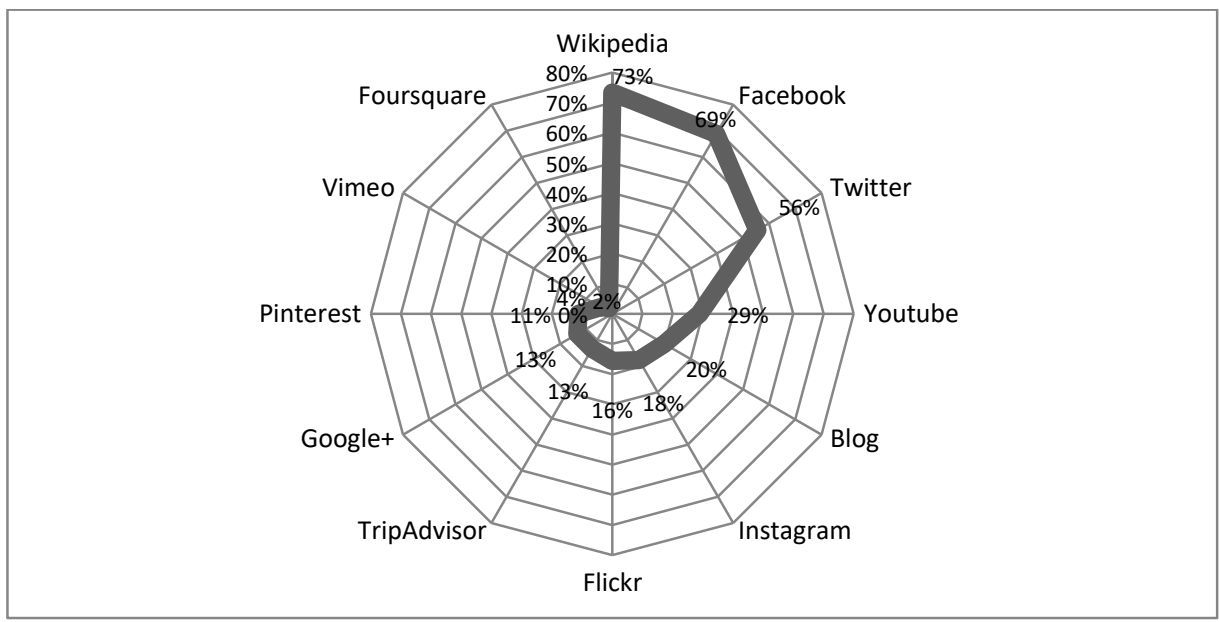

Figure 2. Total or partial presence on social media sites analysed ( $\% 45$ assets)

We also analysed the intensity of the presence of each asset on the different platforms mentioned -both those referring to social networks, and Wikipedia, blogs and Google Arts \& Culture- from the number of platforms on which their institutional URLs were present. 
Table 1. Social platforms employed by the assets

\begin{tabular}{lllllllllll}
\hline Number of platforms & None & $\mathbf{1}$ & $\mathbf{2}$ & $\mathbf{3}$ & $\mathbf{4}$ & $\mathbf{5}$ & $\mathbf{6}$ & $\mathbf{7}$ & $\mathbf{8}$ & $\mathbf{9}$ \\
\hline Percentage of use & $13 \%$ & $31 \%$ & $14 \%$ & $14 \%$ & $13 \%$ & $4 \%$ & $6 \%$ & $3 \%$ & $1 \%$ & $0 \%$ \\
\hline
\end{tabular}

Table 1 shows the usage frequency of these platforms, that is, on how many of them the institutions have included a profile that links to the heritage asset websites. In this regard, attention should be drawn to the fact that over $70 \%$ of the URLs have institutional profiles on $1(31 \%), 2(14 \%), 3(14 \%)$ or, even $4(13 \%)$ social media sites. Furthermore, $14 \%$ of these boast a profile on 5 or more platforms. The heritage asset corresponding to the Alhambra of Granada particularly stands out; with a presence extending to all of the popular media used currently -a total of 10-. Apart from this exception, no asset is found on all of the platforms. This circumstance is undoubtedly influenced by the fact that tools such as TripAdvisor and FourSquare focus mainly on the opinions and comments of their users and/or visitors to the assets in question -their prime objective- rather than the institutions responsible for the management to these assets.

Focusing only the URLs which have an institutional profile on Twitter or Facebook, $79 \%$ of the cases analysed had a presence on both. On the contrary, $18 \%$ only having a Facebook profile and $3 \%$ only a Twitter profile. Besides, we have characterized the presence of cultural heritages which have Facebook and/or Twitter profiles according to the bodies responsible for each heritage asset (table 2). The highest presence on the aforementioned social networks is maintained by institutional URLs of the heritage assets managed by the central or regional administration, either individually or via consortiums between various bodies.

Table 2. Presence of Facebook and/or Twitter profiles

\begin{tabular}{lllllll}
\hline Type of institution & Governmental & Consortium & Municipal Foundation & Religious & Private & Association \\
\hline Facebook/Twitter use & $37 \%$ & $24 \%$ & $26 \%$ & $11 \%$ & $5 \%$ & $5 \%$ \\
\end{tabular}

In relation to the presence of profiles of the sample URLs on the audiovisual networks, the use of video platforms -YouTube and Vimeo- is higher, with $25 \%$ of the institutional websites present on these, compared to $19 \%$ with a profile on the static image based sites Flickr, Instagram and Pinterest. Specifically, 24\% of the sample URLs had a YouTube profile, compared to $3 \%$ for Vimeo. There was only one case where both platforms were used to increase presence on networks.

We studied this phenomenon for 45 assets and analysed whether there were great differences. It was possible to verify that $19 \%$ of the heritage assets recognised as World Heritage in Spain by Unesco have a profile on image-based social networks. $13 \%$ of these assets are represented on Instagram, and $7 \%$ on Pinterest. In relation to the usage frequency of these image-based social media sites (Table 3), it is observed that $57 \%$ of the assets have a profile on one of these platforms, whereas just $7 \%$ use all three.

Table 3. Heritage assets with profiles on image-based social networks

\begin{tabular}{llll}
\hline Number of image platforms & $\mathbf{1}$ & $\mathbf{2}$ & $\mathbf{3}$ \\
\hline Percentage of use & $57 \%$ & $36 \%$ & $7 \%$ \\
\hline
\end{tabular}

Something similar occurs with the video format, where a mere $6 \%$ of heritage assets that make use of video-based social media sites use the two platforms studied, YouTube and Vimeo.

As it was announced in methodology, we explored if web pages of the sample are linked more if they have official profile on any of the different social networks and media of the cultural assets.

In this regard, we carried out a quantitative analysis with the purpose of determining to what extent the inclusion of profiles on platforms of a generalist nature, such as Facebook and Twitter, affect links received (inlinks) by the institutional web pages. To achieve this, we made pertinent queries on the Google search engine and counted the number of permanent links originating from other websites to the URLs in the sample. According to the results obtained, attention may be drawn to the fact that $30 \%$ of the institutional URLs of the heritage assets with a profile on at least one of the two platforms has a high number of links originating from other websites (Figure 3), compared to those that do not make use of these social networks. 


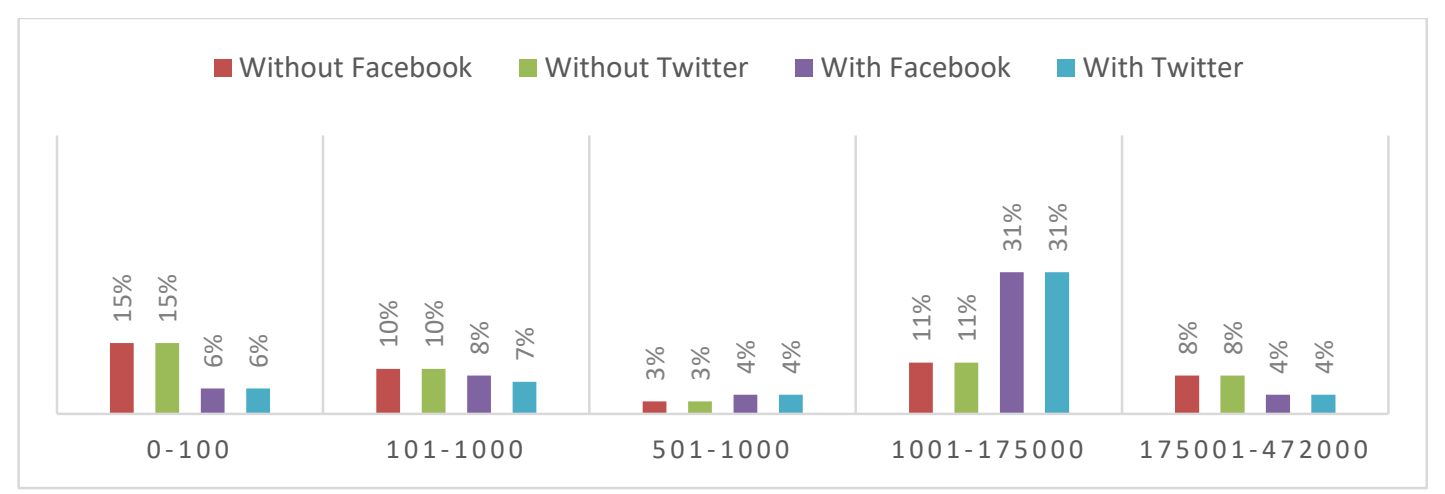

Figure 3. Relationship between inlinks to WebPage and Web 2.0 presence

The blogs analysed reveal differences in design. 55.5\% of the blogs show a traditional design with the periodical inclusion of news and articles, compared to the other $44.4 \%$ which organise content by sections. The content of the blogs is in the Spanish language, with there being only two examples of information in other languages (English, French and Catalan). The greatest proportion of blogs includes images, articles with content and historical data. Others add sections that encourage frequent visits with novelties in actions relating to the heritage asset or events.

Just $12.5 \%$ of the sample URLs corresponding to World Heritage assets have their own blog, compared to $84.5 \%$ which have none. Additionally, $3 \%$ of blogs refer to the institution responsible for the management of the cultural asset, rather than simply the asset itself.

Finally, we have discovered that only $7 \%$ of the assets included in the sample are represented on this Google tool. Each entry displays audio-visual information on the heritage asset in general or on several of the numerous cultural aspects of interest. It links to current or past exhibitions accessible through web resources. Its main information sources are National Libraries and Museums. It also facilitates links to Wikipedia and other resources belonging to the company, such as Google Maps, for information on the location of the heritage asset, or virtual views via Street View. Interest in its geolocation is probably the reason for the cases included in this tool being architectural ensembles within municipalities.

\section{CONCLUSION}

This study confirms the heterogeneous efforts and strategies being carried out by institutions in the social media sphere. However, it also leaves no doubt that there is work left to do in terms of this aspect. Liquid modernity use web 2.0 as usual way to inform and search news. From the perspective of digital humanities, it is worth asking how the dissemination of information on cultural heritage is being implemented. This is the case both regarding the use of Web 2.0 tools and the management of content created for the public. Cultural entities, therefore, should mark the communication and publicity of their content as one of their priorities. In this regard, they are equally obliged to establish and develop a clear, defined strategy for building their digital identity. It is therefore recommendable for those responsible for the dissemination of cultural heritage to make a correct choice regarding the specific communication channel or channels using the language and form of expression adapted accordingly. Accordingly, the choice of social media sites to be used, and even the regular maintenance of the greatest number of them taking into account their specific features, requires professionals dedicated to this task.

In addition, attention must be drawn to the disconnection between the Google Arts \& Culture project and any other social medium without any link that permanently links or connects this resource with the website or with a social profile.

The study carried out highlights a digital identity still lacking for heritage assets as autonomous entities, as they are integrated into the generic profile of the institution responsible for them. As has also been mentioned and in light of these results, it is clear that there is still a long way to go to extend this predisposition to the majority of these managing bodies in developing the communicative task via Web 2.0 social media sites 
effectively, for example taking advantage of audiovisual facilities and social networks, or adopting a multilingual focus in informative resources.

The function of some of these platforms is aimed at tourism in general and cultural tourism (for example TripAdvisor), while others are used as a way of dissemination and interaction with potential visitors. In the future, it is expected to monitor the evolution of the use of these media by these institutions.

We still continue doing research on the development of methodologies that facilitate the automation of data harvesting processes. After carrying out this research we can confirm that the methodology used in this work is adequate for this type of study. Thus, for example, it is capable of being applied in similar analyzes focused on proprietary social networks that do not allow the use of APIs, nor dumping, nor automatic data storage. However, it also has limitations such as the time required to collect and organize the data. Future work will continue to expand this line of research and it is planned to apply automatic information harvesting systems to compare their effectiveness. In addition, the user's perspective will be incorporated regarding the impact caused on them by the communications -both textual and audiovisual- that take place through social media.

\section{REFERENCES}

Bauman, Z. 2003. Modernidad líquida. Fondo de Cultura Económica, México DF

Castelló Martínez. A. 2010. Una nueva figura profesional: el Community Manager. Pangea Vol.1. http://revistapangea.org/ (last consultation: 19 October 2017).

Cohen, J. (Ed.). 1996. Special Issue: Digital Libraries. Communication ACM. Vol. 39, No.11.

García-Santiago, L. and Olvera-Lobo, M.D. 2018. Mexican World Heritage information on the web: Institutional presence and visibility. Information Development, Vol.34, No. 3, pp 261-279.

Google Cultural Institute. 2017. Available at: https://artsandculture.google.com/ (last consultation: 31 August 2020).

Jiménez-Orellana, L.J.2016. Museo y comunicación 2.0. Situación en España. Documentación de las Ciencias de la Información, Vol.39, pp. 177-203.

O'Reilly, Tim. 2005. Web 2.0: compact definition. Available at: http://radar.oreilly.com/2005/10/web-20-compactdefinition.html. (Last consultation: 31 August 2020).

Rojas Castro, A. 2016. Big Data in the Digital Humanities. New Conversations in the Global Academic Contex In: Smart culture. Analysis of digital trends: AC/E Digital culture annual report 2017. DOI: http://dx.doi.org/10.17613/M6434X

Viñarás Abad, $\mathrm{M}$ et al. 2010. La comunicación corporativa de los museos en España a través de la comunicación 3.0: cuatro años de evolución comunicativa en la Red. In: II Congreso internacional comunicación, Salamanca, España. Vol. 3. http://giovannipf.260mb.net/comunicacioncorporativa.pdf?i=1 